\title{
Time Domain Formulation of Self-Excited Forces on Bridge Deck for Wind Tunnel Experiment
}

\author{
Xin Zhang ${ }^{1}$, James Mark William Brownjohn ${ }^{2}$ and Piotr Omenzetter $^{3}$ \\ School of Civil and Environmental Engineering, Nanyang Technological University, 50 Nanyang \\ Avenue, Singapore 639798, Singapore
}

\begin{abstract}
Time domain formulation of the self-excited wind forces on bridge decks employs indicial functions. In bridge aeroelasticity, these functions are obtained by transforming the flutter derivative model to time domain. Studies have suggested, however, that the relative amplitude effect, i.e. the effect of structural oscillation amplitude relative to the amplitude of response to ambient wind, on flutter derivatives needs to be considered. This effect indicates the difference between the two cases, where the pulse response of an elastically supported body is smooth and where the motion is significantly affected by ambient wind forces. The nonlinearity may affect the transformation of flutter derivative model to time domain. An alternative obtaining the time domain formulation for the self-excited force is to treat the self-excited force as a separate dynamic system, so that the relative amplitude effect can be evaluated in more details. In this paper, a self-excited force generation system coupled with the rigid bridge deck system is proposed to overcome the difficulties in the measurement and derivation of the time domain representation of self-excited force on bridge decks. This expression can be linked to a flutter derivative model, and a transform relationship between the two models is suggested.
\end{abstract}

Keywords

Indicial Function, Flutter Derivatives, Self-excited Forces, Time Domain

\footnotetext{
${ }^{1}$ Ph.D. Candidate

${ }^{2}$ Associate Professor

${ }^{3}$ Research Fellow
} 


\section{Introduction:}

\subsection{Previous Work}

The most fundamental task of bridge aeroelasticity lies in the formulation of the self-excited forces, the wind load caused by the movement of the structure. Theodorsen [1] derived the theoretical description of the unsteady aerodynamic forces on the efficient airfoil under sinusoidal motion by employing the reduced frequency dependent Theodorsen's circulation function. Theodorsen and Garrick [2] further extended the work to characterize the non-stationary flow about a wing-aileron-tab combination. Following Sears [3] and Luke and Dengler [4], Edwards [5] showed that these results could be generalized for arbitrary motion. The unsteady aerodynamic forces can thus be formulated by a reduced-frequency dependent aerodynamic influence matrix.

The duality of time and frequency domain formulation of the self-excited wind forces on airfoils was emphasized by Garrick [6]. In time domain, Wagner [7] showed the lift evolution with dimensionless time acting on a theoretical flat airfoil given a step change in angle of attack via an indicial function. Kussner [8] considered the problem of an airfoil with forward flight velocity penetrating a uniform vertical gust of infinite downstream extent and vertical velocity. Sears [3] derived the corresponding oscillatory lift for a gust velocity distribution that is sinusoidal. Jones [9] introduced rational approximations of indicial functions.

In the time domain formulation of self-excited forces on a bridge deck, indicial functions are also adopted by Borri and Hoffer [10] and Brar and Scanlan [11]. Wilde et al. [12], Bucher and Lin [13] and Chen et al. [14], [15] also treated the surrounding airflow as a set of filter-like devices in generating self-excited forces on a bridge by transforming the frequency domain flutter derivatives [16] to time domain for flutter and buffeting analysis of cable supported bridges.

However, the signature turbulence, in the case of efficient airfoils in smooth flow, is intentionally reduced by careful streamlining with notable attention to introduction of a sharp trailing edge. For bluff bodies, the situation is different. The formulation of self-excited forces on civil engineering structures, such as bridge decks, is more experimental than theoretical. The direct measurement of indicial functions, however, is neither easy nor conventional.

Scanlan et al. [17] studied the aeroelastic moment on a bluff bridge deck due to indicial angular movement. The characteristic of corresponding indicial function of a bridge, i.e. the 
rotational aerodynamic damping due to the rotational motion, according to their experiment, is strongly different from those of the corresponding functions of airfoils. It was shown that the relationship between the flutter derivatives and the indicial function is obtained by recognizing that for a sinusoidal motion, the Duhammel integral is of the nature of a Fourier transform [18] and the inverse transform of frequency domain expression should then produce the indicial function. Figure 1 shows the indicial functions with different structural forms. The Jones approximation is for efficient airfoils. The other two curves are experimental measurements from bluff bridge decks. Scanlan et al [17] used the exponential approximation form with two exponential terms to curve fit the experimental data from a truss structure. The measurement of Yoshimura and Nakamura [19] is a direct measurement of indicial aerodynamic moment response of moving bluff prismatic sections of $\mathrm{H}$ or $\mathrm{T}$ type in still air. This curve is scaled to match the magnitude of other curves; it is shown here only qualitatively. Striking differences can be observed from the exponential approximation curves: an initial steep rise from a low negative value to a peak, which "overshoots" the steady state value, then settles down asymptotically. The oscillating component in the curve by Yoshimura and Nakamura is clear and cannot be neglected.

\subsection{Relative Amplitude Effect on the Transformation of Flutter Derivative Model to Time Domain}

The relative amplitude is defined as the ratio of triggered vibration (transient vibration) amplitude of the model to "structural noise" magnitude in the vibration due to the ambient wind excitation. In the numerically simulated figure 2, the dotted line represents a triggered vibration and the continuos line stands for the "noisy background" due to the ambient wind excitation. The relative amplitude can then be defined as $R_{a}=A / \Delta$, where $A$ is the mechanically triggered vibration amplitude and $\Delta$ is an averaging characteristic measurement representing the ambient vibration magnitude. The effect of relative amplitude on flutter derivatives and on the flutter boundary reveals, from the structural point of view, a relationship between the self-excited forces and the "structural vibration noise" due to buffeting forces. If $R_{a} \rightarrow \infty$, the triggered vibration is totally smooth and the effect of turbulence is negligible. If $R_{a} \rightarrow 0$, the triggered vibration is 
severely affected by the ambient dynamic wind load. The latter case can be studied by identifying flutter derivatives from ambient vibration [20].

As have been observed in a study by Zhang and Brownjohn [21] on the identification of flutter derivatives from transient and ambient vibration, the relative amplitude effect on flutter derivatives may not be neglected. Figures 3 show the flutter derivatives of a partially streamlined box girder section (Figure 4). The differences between the results from transient and ambient identification indicate the effects of relative amplitude on flutter derivatives. In the experiments, no turbulence in the oncoming flow was artificially generated by active devices, therefore, the ambient vibration was due to the small turbulence in the oncoming flow and signature turbulence generated by the bluff body itself. Effect of turbulence in the oncoming wind can be studied with the same method, but due to the limitation of experimental devices, was not studied in this research.

If relative amplitude effect is to be considered, it may not be valid to transform the flutter derivative model measured under sinusoidal or exponentially modified oscillation to time domain for general-purpose analysis. An alternative model for the direct identification of interactive forces in time domain from experiments may be favourable so that more complex cases can be studied directly by experiments.

\section{State Space model for Self-Excited Force}

In this section, the self-excited wind load is dealt with as a separate dynamic system. The effort is not to provide a nonlinear model, but to study the interactive forces at different values of relative amplitude.

\subsection{The Model}

The dynamic system of self-excited force is coupled with the elastically supported bridge deck; the rigid body gives excitations to the self-excited force dynamic system and receives feedback from it.

The equations of the sectional model motion are: 


$$
[M] \ddot{x}+[C] \dot{x}+[K] x=f_{\text {sef }}+f_{\text {buff }}
$$

in which $x=\left\{\begin{array}{lll}h & p & \alpha\end{array}\right\}^{T}$ is the displacement vector comprising vertical, lateral and rotational motion, $[M]$ is the structural mass matrix, $[C]$ is the structural damping and $[K]$ is structural stiffness. $f_{\text {buff }}$ is the buffeting force vector due to the fluctuation component in the oncoming flow and signature turbulence generated by the bluff body itself. This term is considered independent of the structural motion. $f_{\text {sef }}$ represents the self-excited forces.

Changing equation (1) to dimensionless time $s$ domain,

$$
s=\frac{U}{B} t
$$

where $s$ is the dimensionless time; $U$ is the wind velocity, $B$ is the width of the bridge deck, one has

$$
\begin{aligned}
& x^{\prime \prime}(s)+\left(\frac{B}{U}\right)[M]^{-1}[C] x^{\prime}(s)+\left(\frac{B}{U}\right)^{2}[M]^{-1}[K] x(s) \\
& =\left(\frac{B}{U}\right)^{2}[M]^{-1}\left(f_{\text {sef }}+f_{\text {buff }}(s)\right)
\end{aligned}
$$

where 'and " are the first and second derivatives of corresponding variable with respect to dimensionless time $s$, respectively.

The state space form of equation (1) is:

$$
\begin{aligned}
& X^{\prime}(s)=A_{s} X(s)+B_{s}\left(f_{\text {sef }}(s)+f_{\text {buff }}(s)\right) \\
& Y(s)=C_{s} X(s)
\end{aligned}
$$

with state vector

$$
X(s)=\left\{h(s) \quad p(s) \quad \alpha(s) \quad h^{\prime}(s) \quad p^{\prime}(s) \quad \alpha^{\prime}(s)\right\}^{T}
$$

and the state matrix is

$$
A_{s}=\left[\begin{array}{cc}
0 & I \\
-\left(\frac{B}{U}\right)^{2}[M]^{-1}[K] & -\left(\frac{B}{U}\right)[M]^{-1}[C]
\end{array}\right]
$$

The input matrix for self-excited forces and buffeting forces vector is

$$
B_{s}=\left[\left(\frac{B}{U}\right)^{2}[M]^{-1}\right]
$$


and the output matrix is

$$
C_{s}=\left[\begin{array}{llllll}
1 & 0 & 0 & 0 & 0 & 0 \\
0 & 1 & 0 & 0 & 0 & 0 \\
0 & 0 & 1 & 0 & 0 & 0
\end{array}\right] .
$$

Similarly, the state space formulation for the flutter derivative model is obtained by using reduced frequency dependent matrices $[C]_{\text {aero }}$ and $[K]_{\text {aero }}$ :

$$
f_{\text {sef }}(t)=[C]_{\text {aero }} \dot{x}(t)+[K]_{\text {aero }} x(t) \text {. }
$$

Therefore equation (4) can be rewritten as

$$
\begin{aligned}
& X^{\prime}(s)=A_{s}^{f} X(s)+B_{s} f_{\text {buff }}(s) \\
& Y(s)=C_{s} X(s)
\end{aligned}
$$

where

$$
\begin{aligned}
& A_{s}^{f}=\left[-\left(\frac{B}{U}\right)^{2}[M]^{-1}[K]_{\text {eff }}-\left(\frac{B}{U}\right)[M]^{-1}[C]_{\text {eff }}\right] \\
& {[K]_{\text {eff }}=[K]-[K]_{\text {eero }} \text { and }} \\
& {[C]_{\text {eff }}=[C]-[C]_{\text {aero }}}
\end{aligned}
$$

The dynamic system of self-excited force is also formulated by state space equations in dimensionless time domain. One fundamental question is how to determine the input of the system. It can be argued that the dimensionless time derivative of the rigid body state vector can be considered as the input "force" of the self-excited force dynamic system.

A proof is due to Bisplinghoff and Ashley [22]. They have pointed out that indicial response functions corresponding to lift and moment due, respectively, to step changes in effective angle of attack and effective rate of change of angle of attack, should be used to formulate the unsteady aeroelastic force on airfoil. Lin and Yang [23] held the same idea and suggested impulse response functions of the self-excited forces due to velocity and acceleration of the rigid body, respectively. It is justified to argue that the time derivative of rigid body state vector, i.e. $\frac{d}{d s} X(s)$, can be used as the input of the concerned system.

The self-excited force system may be modeled by a linear state space model, with order up to experimental determination, in dimensionless time domain: 


$$
\begin{aligned}
& f^{\prime}(s)=F f(s)+B_{f} \frac{d}{d s} X(s) \\
& f_{s e f}(s)=C_{f} f(s)
\end{aligned}
$$

in which $f(s)$ is the $n \times 1$ state vector of self-excited force system,

$f^{\prime}(s)$ is the dimensionless time derivative of $f(s)$ with respect to dimensionless time,

$F$ is the $n \times n$ square state matrix,

$B_{f}$ is the $n \times 6$ input matrix,

$C_{f}$ is the $3 \times n$ output matrix.

The value of $n$, i.e. the system order, is to be determined from experimental data.

The coupled system governed by equations (4) and (14) can be expressed in the form of simulation diagram showed in figure 5 . In the diagram, the self-excited forces and the bridge deck system are referred to as SEF and BDS, respectively. The SEF takes the dimensionless time derivative of the BDS state vector as its input and returns the self-excited forces as output to the BDS as a part of its input.

\subsection{Relation to Flutter Derivative Model}

The duality of indicial functions and flutter derivatives has been emphasized by other researchers such as Scanlan [24]. The transform relationship between time domain model and flutter derivative model is important.

When there is exponentially modified or pure sinusoidal motion of the bridge deck section, the self-excited forces can be described by flutter derivative model [25]. For ambient response, we consider the self-excited wind forces due to a small impulse response of the bridge deck in wind. As shown in the Appendix, this is equivalent to considering self-excited wind forces due to the output covariance of the ambient vibration of the body [20][26].

Let the flutter derivative matrix be 


$$
[\mathrm{H}(K)]=\left[\begin{array}{cccccc}
\frac{K^{2}}{B} H_{4}^{*} & \frac{K^{2}}{B} H_{6}^{*} & K^{2} H_{3}^{*} & \frac{K}{B} H_{1}^{*} & \frac{K}{B} H_{5}^{*} & K H_{2}^{*} \\
\frac{K^{2}}{B} P_{4}^{*} & \frac{K^{2}}{B} P_{6}^{*} & K^{2} P_{3}^{*} & \frac{K}{B} P_{1}^{*} & \frac{K}{B} P_{5}^{*} & K P_{2}^{*} \\
\frac{K^{2}}{B} A_{4}^{*} & \frac{K^{2}}{B} A_{6}^{*} & K^{2} A_{3}^{*} & \frac{K}{B} A_{1}^{*} & \frac{K}{B} A_{5}^{*} & K A_{2}^{*}
\end{array}\right]
$$

The self-excited forces model is rewritten in a matrix form:

$$
f_{s e f}(s)=\frac{1}{2} \rho U^{2} B[\mathrm{H}(K)] X(s)
$$

in which $f_{s e f}=\left\{\begin{array}{lll}L_{a e} & D_{a e} & M_{a e}\end{array}\right\}^{T}$ is the self-excited lift drag and moment forces. $\rho$ is the air density, $K=\frac{B \omega}{U}$ is the reduced frequency and $H_{i}^{*}, P_{i}^{*}, A_{i}^{*}, i=1, \ldots 6$ are flutter derivatives.

It seems easier to adopt a different format by using Duhammel integral to represent the "fluid memory". The "force" term is the time derivative of rigid body state vector:

$$
f_{s e f}=\frac{1}{2} \rho U^{2} B \int_{-\infty}^{s}[\Phi(s-\sigma)] \frac{d}{d \sigma} X(\sigma) d \sigma
$$

in which

$$
\frac{1}{2} \rho U^{2} B[\Phi(s)]=C_{f} e^{F s} B_{f},
$$

is the pulse response function of self-excited force dynamic system.

The relationship between the flutter derivative model and the dimensionless time domain model can be developed as follows: substitute the rigid body state space equation (10) into (17) and equating to (16):

$$
[H(k)] X(s)=\int_{-\infty}^{s}[\Phi(s-\sigma)]\left\{A_{s}^{f} X(\sigma)+B_{s} f_{b u f f}(\sigma)\right\} d \sigma
$$

Change the integration variable $\sigma$ to $\tau=s-\sigma$

$$
[H(k)] X(s)=\int_{0}^{\infty}[\Phi(\tau)]\left\{A_{s}^{f} X(s-\tau) d \tau+\int_{0}^{\infty}[\Phi(\tau)] B_{s} f_{\text {buff }}(s-\tau) d \tau\right.
$$

Taking Laplace transform and using convolution property, we have

$$
[H(k)] \bar{X}(k)=[\bar{\Phi}]\left\{A_{s}^{f} \bar{X}(k)+B_{s} \bar{f}_{\text {buff }}(k)\right\}
$$

in which, an over bar denotes Laplace transform. 
For the rigid body state space equation (10), because the buffeting force is considered as process noise, which is white around the reduced natural frequency, its magnitude should be much smaller than that of the state vector. Therefore

$$
A_{s}^{f} \bar{X}(k)>>B_{s} \bar{f}_{b u f f}(k) .
$$

The covariance function equation (A.6) corresponds to the noise free case, i.e. $\bar{f}_{\text {buff }}(k)=0$.

Therefore, in both cases equation (21) yields

$$
[H(k)] \bar{X}(k) \approx[\bar{\Phi}] \cdot A_{s}^{f} \bar{X}(k)
$$

The general relationship between the flutter-derivative matrix and the transfer function matrix of the self-excited force system is:

$$
[\bar{\Phi}(k)] \approx[\mathrm{H}(k)]\left(A_{s}^{f}\right)^{-1}
$$

Figure 6 shows the matrix $[\bar{\Phi}(k)]$ obtained from flutter derivatives shown in Figure 3. The $[\bar{\Phi}(k)]$ matrix so identified must be real due to the fact that the flutter derivative matrix is real.

The other observation is that the transformation dependents on matrix $\left(A_{s}^{f}\right)^{-1}$, which, consists of aeroelastic coupling due to the self-excited forces in addition to the structural properties. If the motion is decoupled, e.g. sinusoidal rotational motion, the system matrix must have the following format:

$$
A_{s}^{f}=\left[\begin{array}{cc}
0 & I \\
-K^{2} & 0
\end{array}\right],
$$

so that the aeroelastic matrix is

$$
[\mathrm{H}]=\left\lfloor\begin{array}{ll}
K^{2} A_{3}^{*} & K A_{2}^{*}
\end{array}\right\rfloor,
$$

therefore

$$
\left[\begin{array}{ll}
\bar{\Phi}_{M \alpha^{\prime}} & \bar{\Phi}_{M \alpha^{\prime \prime}}
\end{array}\right]=[H]\left[\mathrm{A}_{\mathrm{s}}^{\mathrm{f}}\right]_{\mathrm{s}}^{-1}=\left[\begin{array}{ll}
K A_{2}^{*} & -A_{3}^{*}
\end{array}\right] .
$$

Because the state vector is

$$
X(s)=\left\{x_{0} e^{i K s} \quad i K x_{0} e^{i K s}\right\}^{T},
$$

we define impulse function for moment due to the rotational movement as

$$
\bar{\Phi}_{M \alpha}=\bar{\Phi}_{M a^{\prime}}+i K \bar{\Phi}_{M \alpha^{\prime \prime}}
$$

so that

$$
\bar{\Phi}_{M \alpha}=K\left[A_{2}^{*}-i A_{3}^{*}\right]
$$


This single DOF transform relationship was also suggested by Brar and Scanlan [12].

\section{$3 \quad$ Suggestions and Conclusions}

One of the possible issues concerning the relative amplitude effect on the measurement of flutter derivatives is as follows. In transient vibration tests, the triggered vibration is clearly larger than the ambient vibration at the beginning, but decays very fast into the ambient vibration envelope. When the vibration starts, the relative amplitude effect is negligible but it is not at the end. The identification of flutter derivatives corresponding to the free decay vibration actually deals with a time-varying phenomenon. On the other hand, in the conventional forced vibration tests, the ambient vibration is "screened out" by the rigid forcing devices and does not present in the tests. By using the dimensionless time domain model proposed in this paper, it is possible to study the effect in more detail.

The practice may need non-contact active drivers (e.g. electro-magnetic force driver) to force the model so that the model, undergoing a "noisy" forced sinusoidal motion, is not constrained by the forcing device as it is in the conventional forced vibration tests. By choosing the magnitude of the driving force, the experiment can be done with a set of controlled relative amplitude.

Because the ambient vibration "noise" level in the tests is the major topic of concern, the forced vibration may be considered to contain other frequency components besides the natural frequency of the suspension system. In view of this, a time domain model of self-excited forces may be an alterative for further study of the relative amplitude effect. 


\section{Appendix: The Correlation Function of the Output Signal}

The correlation function of the output signal is:

$$
\begin{gathered}
\operatorname{Cov}(Y(s+\delta), Y(s))=E\left[C\left\{e^{A_{s}^{f} \delta} X(s)+\int_{s}^{s+\delta} e^{A_{s}^{f}(s+\delta-\tau)} B_{s} f_{\text {buff }}(\tau) d \tau\right\} X^{T}(s) C^{T}\right] \\
=C e^{A_{s}^{f} \delta} \operatorname{Cov}_{s} C^{T}+E\left[C \int_{s}^{s+\delta} e^{A_{s}^{f}(s+\delta-\tau)} B_{s} f_{\text {buff }}(\tau) d \tau X^{T}(s) C^{T}\right]
\end{gathered}
$$

in which

$$
\operatorname{Cov}_{s}=E\left[X(s) X^{T}(s)\right]
$$

and $E[\bullet]$ is the mathematical expectation.

If the condition is met that the system is subjected to white noise, and the fluctuating buffeting force containing no memory of the bridge deck vibration history, considering this is a centered process, $E[Y(s)]=C E[X(s)]=0$, the second term in equation (A.1) vanishes, i.e.,

$$
\left.C E\left[\int_{s}^{s+\delta} e^{A_{s}^{f}(s+\delta-\tau)} B_{s} f_{b u f f}(\tau) d \tau\right\}\right] E\left[X^{T}(s)\right] C^{T}=0
$$

It gives rise to

$$
\operatorname{Cov}(Y(s+\delta), Y(s))=C e^{A_{s}^{f} \delta} B_{c o v}
$$

where

$$
B_{\text {cov }}=\operatorname{Cov}_{s} C^{T} \text {, }
$$

This gives rise to a linear dynamic system:

$$
\begin{aligned}
& X^{\prime}(s)=A_{s}^{f} X(s)+B_{c o v} \delta \\
& Y(s)=C X(s)
\end{aligned}
$$




\section{Reference}

[1] Theodorsen, T., (1935)

General theory of aerodynamic instability and mechanization of flutter

NACA Rept. 496, 1935

[2] Theodorsen, T. Garrick, I. E. (1943)

Non-stationary flow about a wing-aileron-tab combination including aerodynamic balance

NACA. Rept. 736, 1943

[3] Sears, W. R., 1940

Operational methods in the theory of airfoils in nonuniform motion

Journal of the Franklin Institute Vol. 230, No. 1, July 1940

[4] Luke, Y. and Dangler, M. A. 1951

Tables of Theodorsen circulation function for generalized motion

Journal of the aeronautical sciences July 1951, pp. $478-483$

[5] Edwards, J. W. 1977

Unsteady aerodynamic modeling and active aeroelastic control

SUDDAR 504 Stanford University, Feb. 1977

[6] Garrick, I.E. (1938)

On some reciprocal relations in the theory of nonstationary flows

NACA Technical Report 629, 1983

[7] Wagner, H. (1925)

Uber die entstehung des dynamischen auftriebes von tragflugeln

Zeit. Angew. Math. U. Mech., 5(1), 17-35 (in German)

[8] Kussner, H. G., (1936)

Zusammenfassender bericht uber den instantionaren auftried von flugeln

Luftfahrt-Forschung, 13,410-424

[9] Jones, R.T. 1941

The unsteady lift of a wing of finite aspect ratio

NACA Rept. 681, 1941

[10] Borri, C. and Hoffer, R. (2000)

Aeroelastic wind forces on flexible girders 
Meccanica, 35 (10) (2000) 1-15

[11] Brar, P.S. and Scanlan, R.H. (1996)

Numerical calculation of flutter derivatives via indicial functions,

Journal of Fluids and Structures 10(1996), 337-351

[12] Wilde, K. Fujino, Y and Masukawa, J. (1996)

Time domain modeling of bridge deck flutter

Structural Engineering/Earthquake Engineering, JSCE 13 (2) (1996) 93s-104s

[13] Bucher, C. G., and Lin, Y. K. (1988).

Stochastic stability of bridges considering coupled modes.

Journal of Engineering Mechanics, ASCE, 114(12), 1998, 2055-2070

[14] Chen, X. Z., (2000 a)

Time domain flutter and buffeting response analysis of bridges

Journal of Engineering Mechanics, ASCE, Vol. 126 No.1, Jan. 2000, 7-16

[15] Chen, X. Z., (2000 b)

Aerodynamic coupling effects on flutter and buffeting of bridges

Journal of Engineering Mechanics, ASCE, Vol. 126 No.1, Jan. 2000, 17-26

[16] Scanlan, R.H.; Tomko, J. J. (1971)

Airfoil and bridge deck flutter derivatives

Journal of Engineering Mechanics ASCE 97(6) 1971 1717-1737

[17] Scanlan, R. H., Beliveau, J. G. and Budlong, K. S. 1974

Impulse aerodynamic functions for bridge decks

Journal of Engineering Mechanics Division ASCE, Vol. 100, 1974, 657-672

[18] Sabzevari, A., (1971)

Aerodynamic response of suspension bridge to wind gust

Proceedings, $3^{\text {rd }}$ International Conference on Wind Loads on Buildings and Structures, Tokyo, Japan, Oct. 6 10, 1971, Paper IV-37

[19] Yoshimura,T. and Nakamura, Y.

On the indicial aerodynamic moment response of bridge deck sections

Proceedings, Fifth international conference on wind Engineering, Fort Collins, Colo., July, 1979, 877-885

[20] Bogunovic-Jakobsen, J. (1995) 
Fluctuating wind load and response of a line-like engineering structure with emphasis on motion-induced wind forces

Doktor ingenioravhandling, 1995:62

Institutt for Konstruksjonsteknikk Trondheim

[21] Zhang, X and Brownjohn, JWM, 2002

Effect of relative Amplitude and lateral derivatives on flutter I:

Experiment detecting nonlinearity in self-excited forces

Submitted to JWEIA

[22] Bisplinghoff, R. L., and Ashley, H.

Principle of aeroelasticity

John Wiley and Sons, Inc., New York, N. Y., 1962

[23] Lin, Y. K. and Yang, J. N. 1983

Multimode bridge response to wind excitations

Journal of Engineering Mechanics, ASCE, Vol. 109, No. 2, Apr. 1983, 586-603

[24] Scanlan, R. H. (2000)

Motion-related body-force functions in two-dimensional low-speed flow

Journal of Fluids and Structures 14 (2000), 49-63

[25] Jain, A; Jones, N. P., Scanlan, R. H. (1996)

Coupled flutter and buffeting analysis of long-span bridges

Journal of Structural Engineering, ASCE, Vol. 122 No.7, July, 1996, 716-725

[26] Hoen, C.; Moan, N.; Remseth, S. (1993)

System identification of structures exposed to environmental loads

Structural Dynamics-EURODYN'93, Moan et al. (eds) Balkema, Rotterdam. 


\section{Nomenclature}

A

$A_{m}^{*}, H_{m}^{*}, P_{m}^{*}$

$A_{s}, A_{s}^{f}$

B

$B_{f}, B_{s}, B_{c o v}$

$[C],[C]_{\text {aero }},[C]_{\text {effect }}$

$C_{f}$

$C_{s}$

Cov

$f(s)$

$f_{\text {buff }}$

$f_{\text {sef }}$

F

$[H(k)]$

K

$[K],[K]_{\text {aero }},[K]_{\text {effect }}$

$[M]$

$M_{a e}$

$R_{a}$

$s$

$U$

$X(s)$ amplitude of triggered vibration

flutter derivatives

state matrix of rigid body system

deck width

input matrix of SEF, rigid body and covariance dynamics system

structural, aeroelastic and effective damping matrix

output matrix of SEF system

output matrix of rigid body system

covariance estimation

State vector of SEF

buffeting force

self-excited forces

state matrix of SEF system

flutter derivative matrix

reduced frequency

structural, aeroelastic and effective stiffness

structural mass matrix

aeroelastic moment

relative amplitude

dimensionless time

wind speed

state vector of rigid body motion 
$Y(s) \quad$ output vector of rigid body state space model

$\Delta$ averaging character measurement of ambient vibration amplitude

$[\Phi(k)],[\bar{\Phi}(k)] \quad$ pulse response function of self-excited force dynamic system 


\section{List of Figures}

Figure 1 Indicial Functions of Different Kinds

Figure 2 Definition of Relative Amplitude

Figure 3a Two Degree of Freedom Flutter Derivatives $\left(H_{i}^{*}\right)$

Figure $3 \mathrm{~b} \quad$ Two Degree of Freedom Flutter Derivatives $\left(A_{i}^{*}\right)$

Figure 4 Streamlined Box Girder Model (mm)

Figure 5 Simulation Diagram of the Time Domain Model

Figure 6a $\quad[\bar{\Phi}(k)]$ Matrix via Flutter Derivatives (Transient Vibration)

Figure 6b $\quad[\bar{\Phi}(k)]$ Matrix via Flutter Derivatives (Ambient Vibration) 


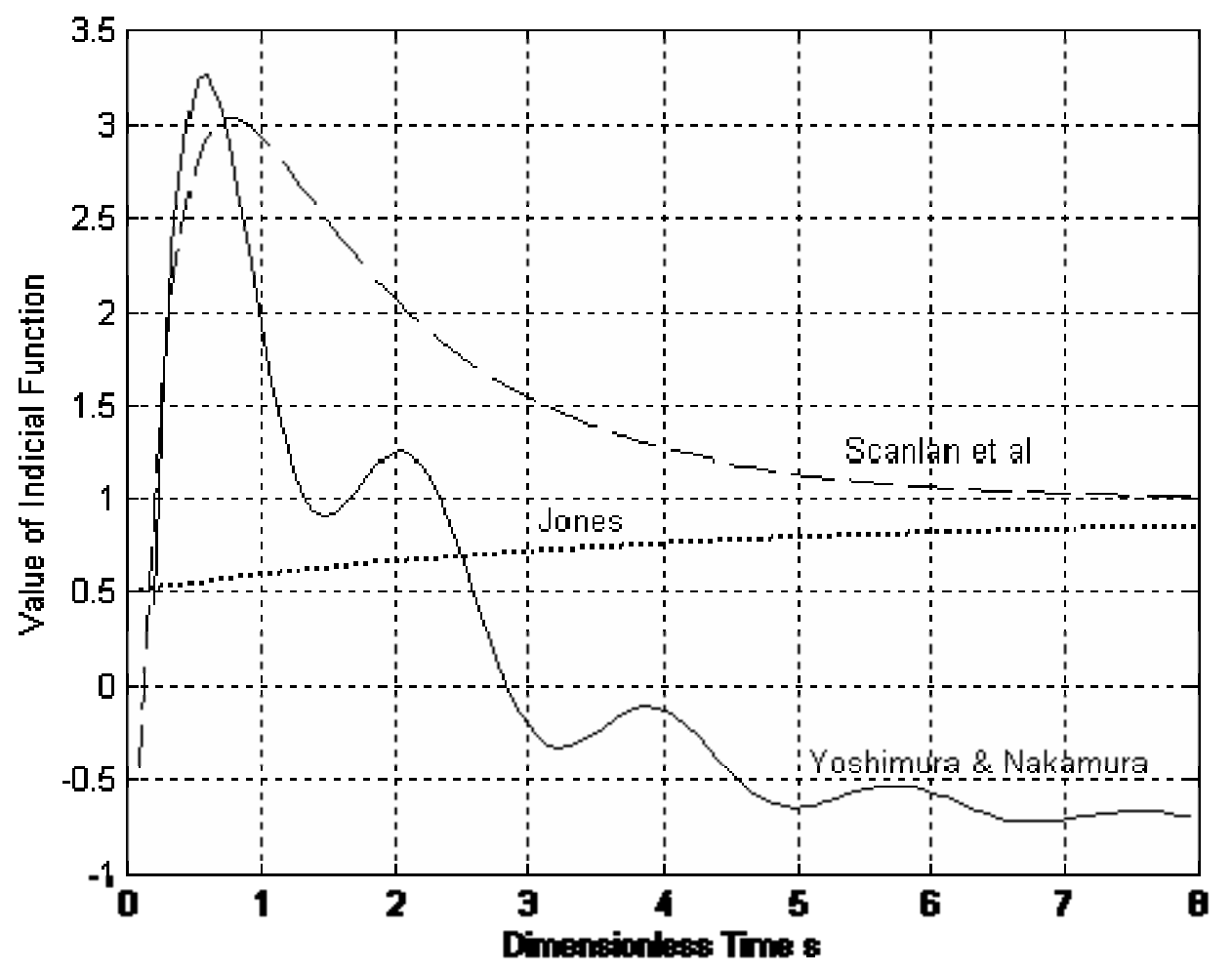

Figure 1 Indicial Functions of Different Kinds 


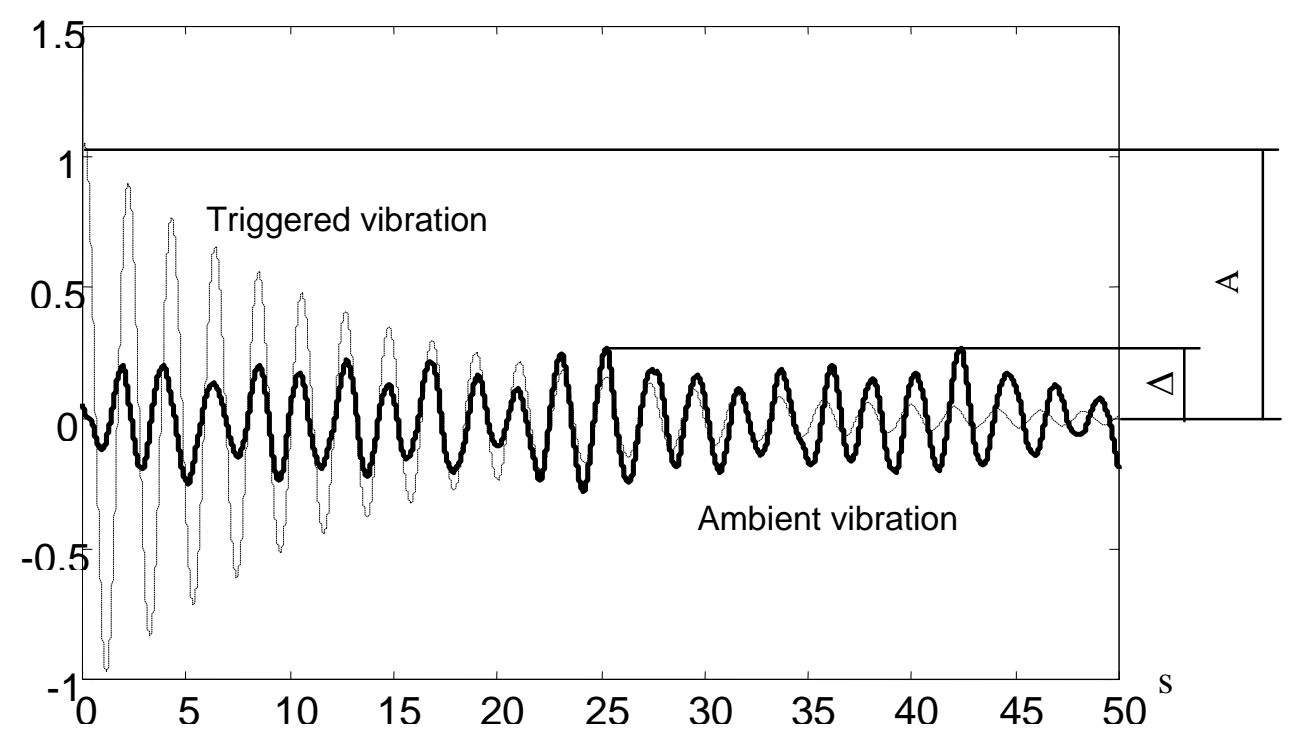

Figure 2 Definition of Relative Amplitude 

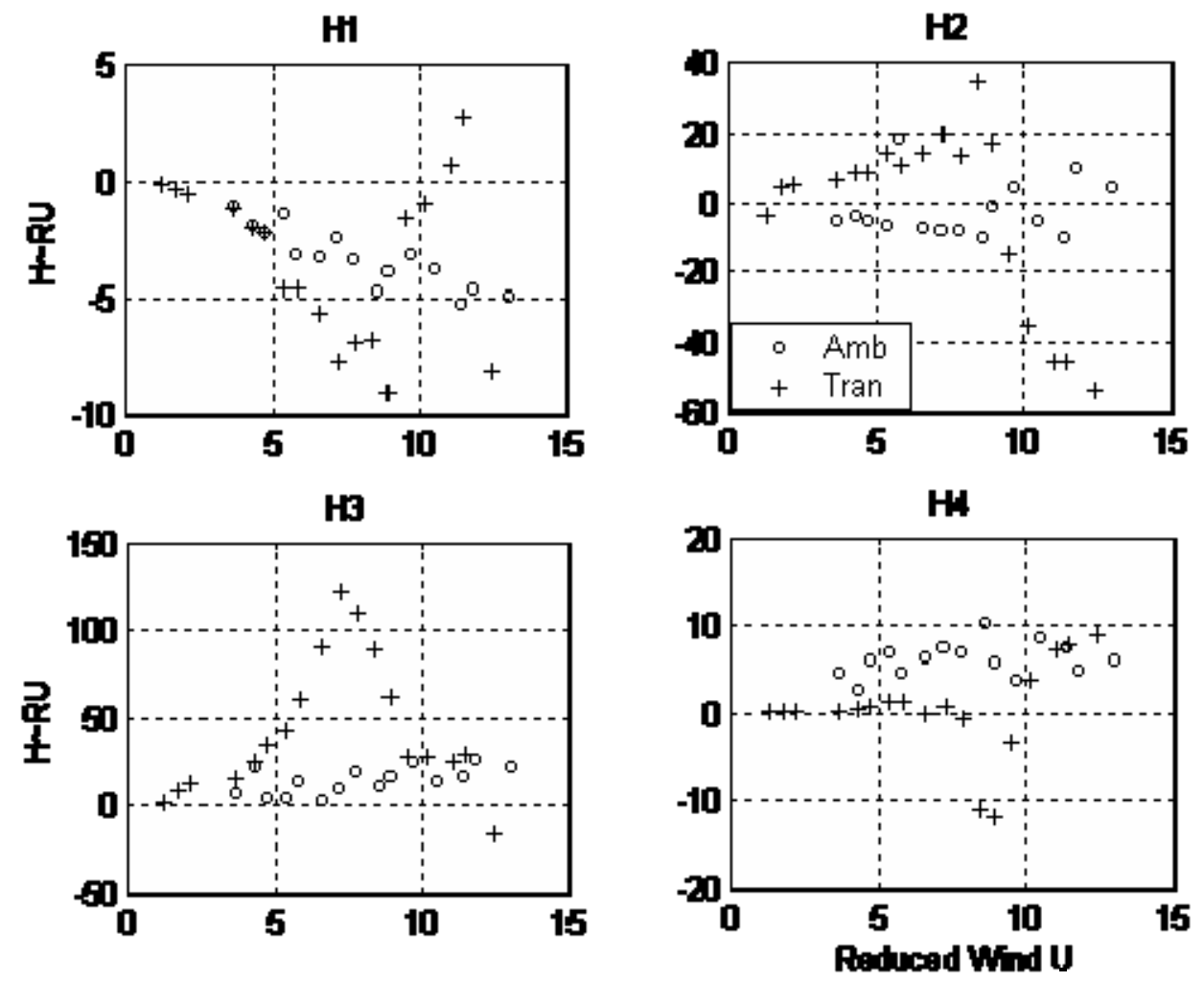

Figure 3a Two Degree of Freedom Flutter Derivatives $\left(H_{i}^{*}\right)$ 

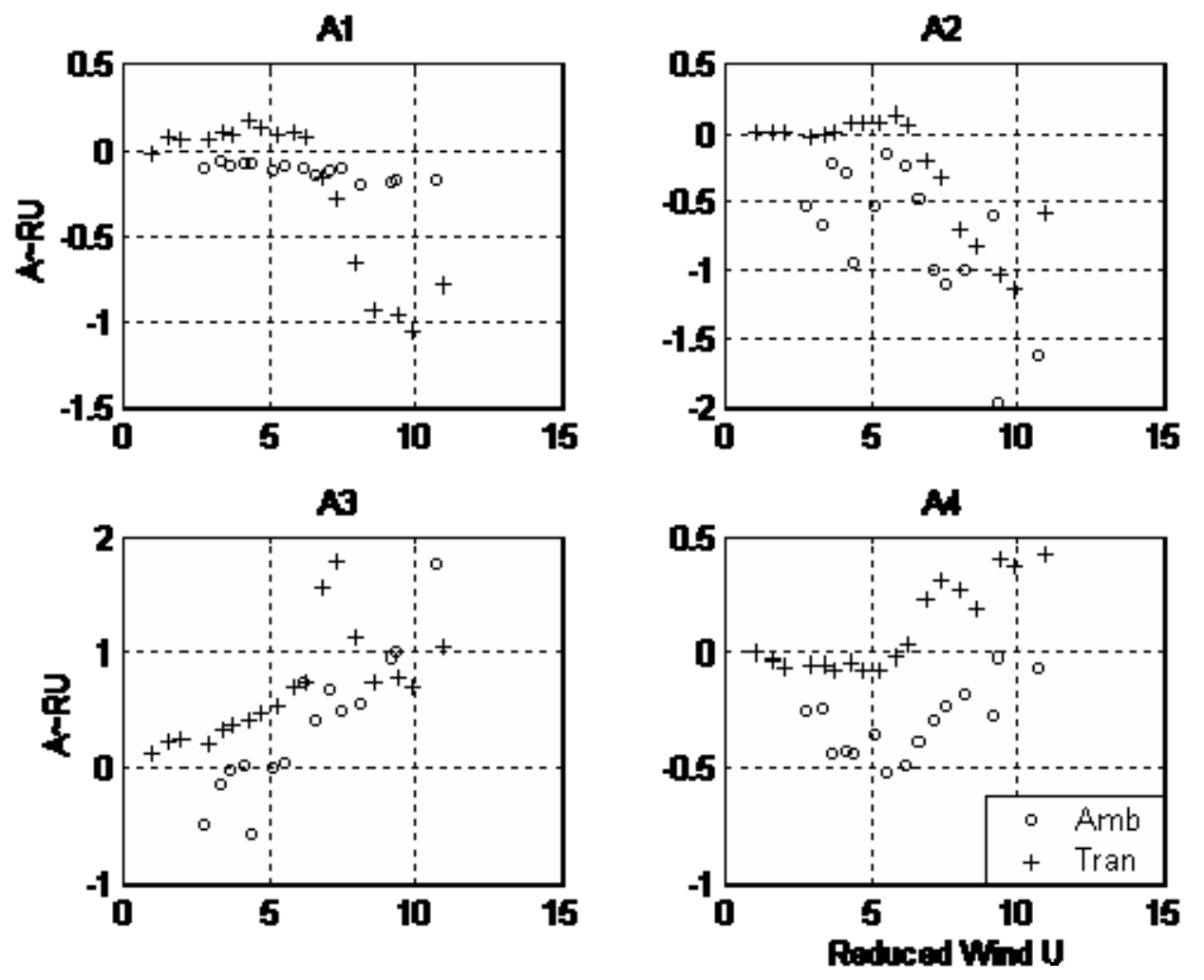

Figure 3b Two Degree of Freedom Flutter Derivatives $\left(A_{i}^{*}\right)$ 


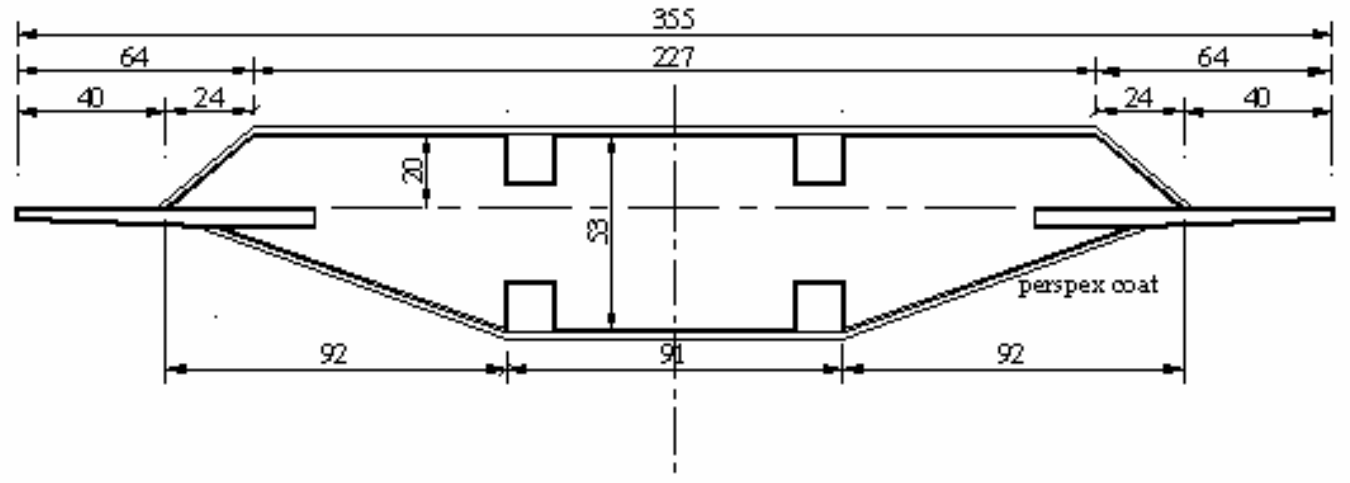

Figure 4 Streamlined Box Girder Model (mm) 


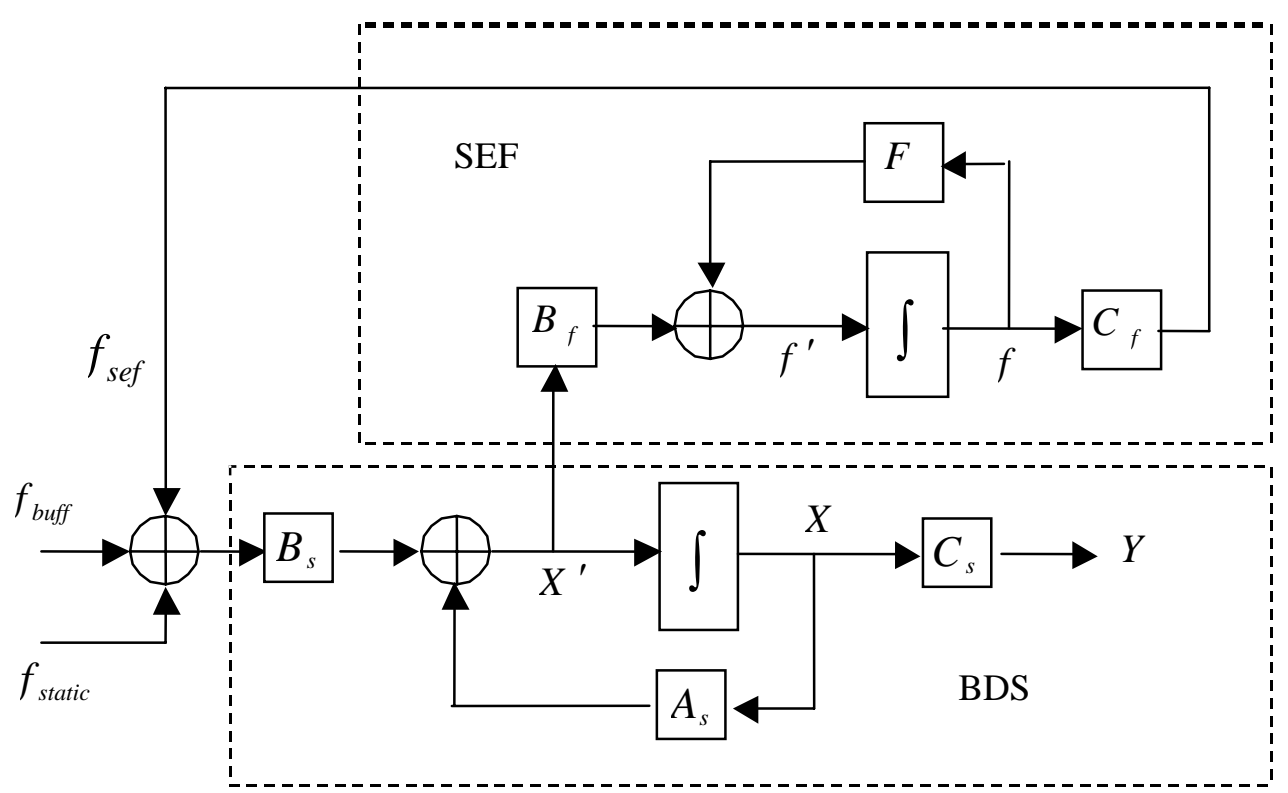

Figure 5 Simulation Diagram of the Time Domain Model 

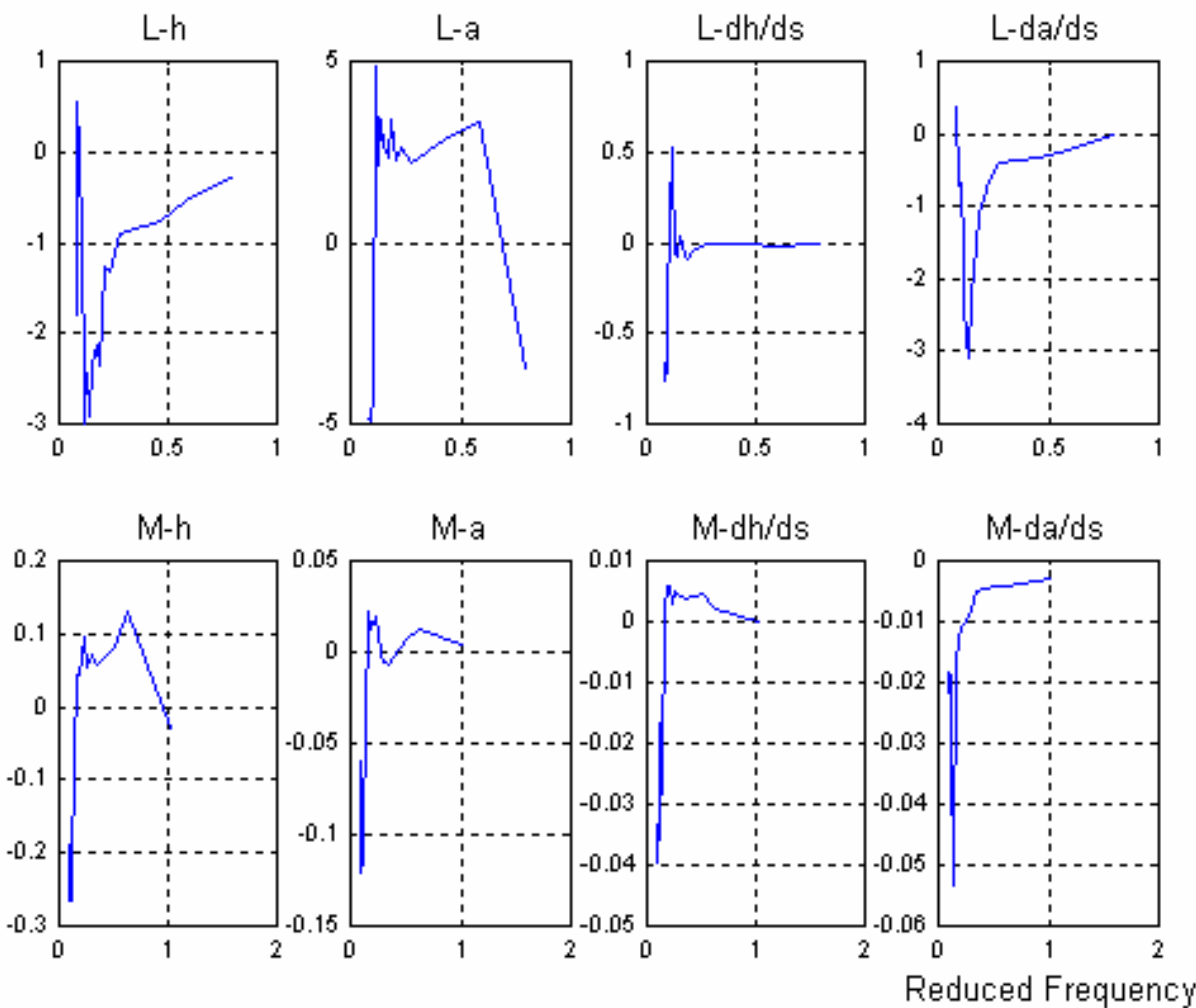

Figure 6a $[\bar{\Phi}(k)]$ Matrix via Flutter Derivatives (Transient Vibration) 

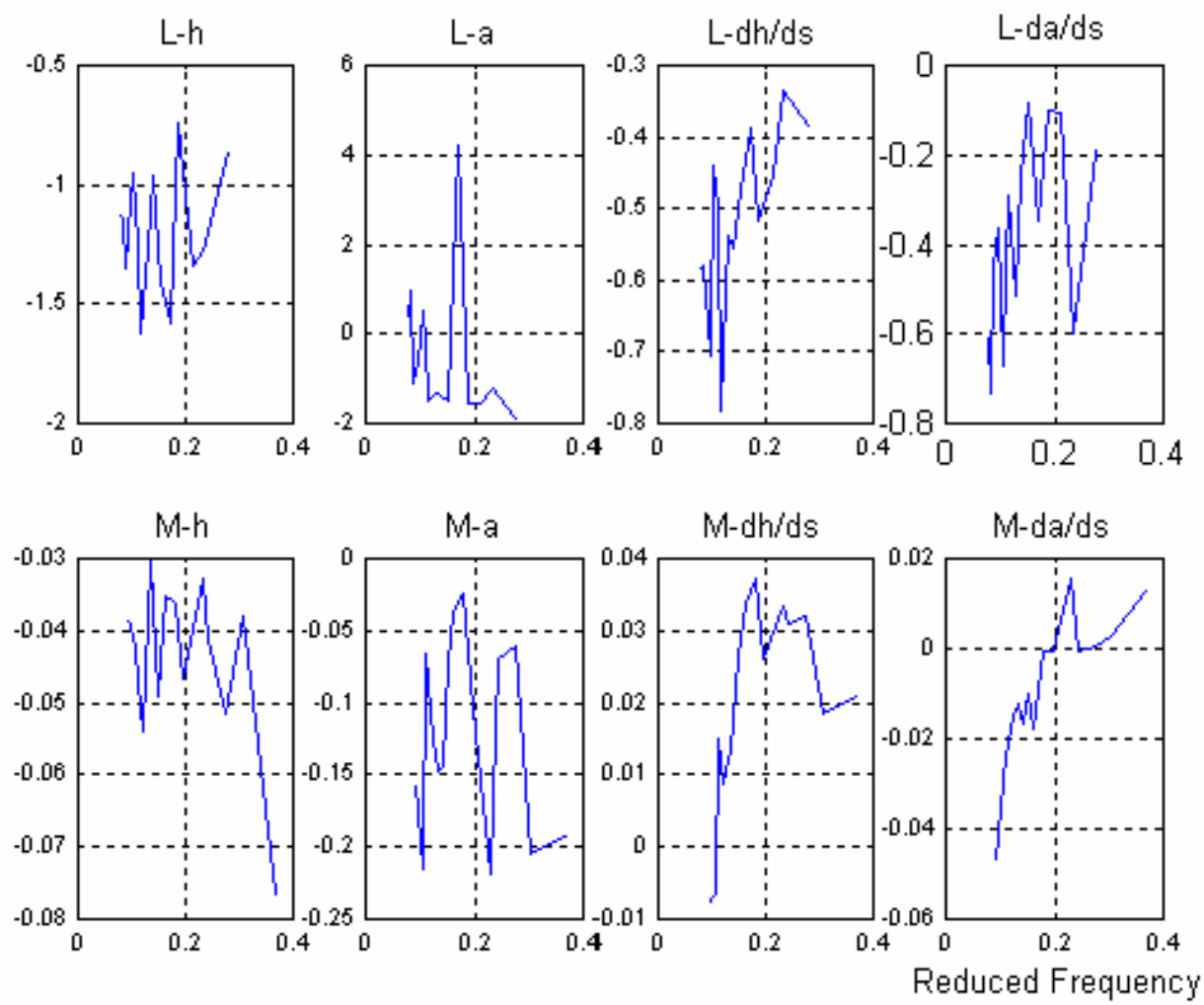

Figure 6b $[\bar{\Phi}(k)]$ Matrix via Flutter Derivatives (Ambient Vibration) 